\title{
Functional Inequalities and Hamilton-Jacobi Equations in Geodesic Spaces
}

\author{
Zoltán M. Balogh • Alexandre Engulatov • \\ Lars Hunziker • Outi Elina Maasalo
}

Received: 23 September 2009 / Accepted: 29 March 2011 / Published online: 28 April 2011

(C) Springer Science+Business Media B.V. 2011

\begin{abstract}
We study the connection between the $p$-Talagrand inequality and the $q$-logarithmic Sololev inequality for conjugate exponents $p \geq 2, q \leq 2$ in proper geodesic metric spaces. By means of a general Hamilton-Jacobi semigroup we prove that these are equivalent, and moreover equivalent to the hypercontractivity of the Hamilton-Jacobi semigroup. Our results generalize those of Lott and Villani. They can be applied to deduce the $p$-Talagrand inequality in the sub-Riemannian setting of the Heisenberg group.
\end{abstract}

Keywords Logarithmic-Sobolev inequalites • Talagrand inequalites • Hamilton-Jacobi semigroup • Poincaré inequalities • Geodesic metric space • Metric-measure space

Mathematics Subject Classifications (2010) Primary 70H20 • 49L99; Secondary 36C05 - 47D06

Z. M. Balogh was supported by the Swiss Nationalfond, EC Project GALA: "Sub-Riemannian geometric analysis in Lie groups," and ERC Project HCAA, "Harmonic and complex analysis and applications", while A. Engulatov and O. E. Maasalo were supported by the EC Project GALA: "Sub-Riemannian geometric analysis in Lie groups."

Z. M. Balogh · A. Engulatov $(\bowtie)$. O. E. Maasalo

Institute of Mathematics, University of Bern, Sidlerstrasse 5, 3012 Bern, Switzerland e-mail: alexandre.engulatov@gmail.com

Z. M. Balogh

e-mail: zoltan.balogh@math.unibe.ch

O. E. Maasalo

e-mail:maasalo@math.unibe.ch

L. Hunziker

Department of Mathematics, University of Technology, PO Box 123, Broadway, Sydney,

NSW 2007, Australia

e-mail: lars.hunziker@uts.edu.au 


\section{Introduction}

The main purpose of the present paper is to study relations between functional inequalities on proper geodesic metric measure spaces. More precisely, we prove that under some additional assumption on the space, the $q$-logarithmic Sobolev inequality and the $p$-Talagrand inequality are equivalent for the conjugate exponents $p \geq 2$ and $q \leq 2$. Transportation inequalities, such as the Talagrand inequality, first appeared in the works of Talagrand and Marton [24, 25, 30]. Our result generalizes the recent results of Lott and Villani, who considered similar questions in the quadratic case when $p=q=2$; see [22]. As in [22], the Hamilton-Jacobi infimum convolution operator plays a crucial role in our approach. This idea goes back to the work of Bobkov et al. [4]. They proved that in Euclidean spaces a measure $\mu$ which is absolutely continuous with respect to the Lebesgue measure satisfies the classical logarithmic Sobolev inequality if and only if the Hamilton-Jacobi semigroup associated to the quadratic infimum-convolution operator is hypercontractive. Gentil and Malrieu generalized this to a broader class of logarithmic Sobolev inequalities; see [12]. In [14] Gozlan et al. establish the equivalence, in a smooth setting, between the Talagrand inequality and the so-called restricted logarithmic Sobolev inequality for a certain class of cost functions.

Lott and Villani applied the same strategy on a compact length space $(X, d)$ equipped with a Borel probability measure $\mu$ to prove the following. If the space supports a local Poincaré inequality and the measure is doubling, then the quadratic logarithmic Sobolev inequality implies the quadratic Talagrand inequality with the same constant. In both proofs, [4] and [22], it is crucial that the infimum-convolution semigroup solves the Hamilton-Jacobi equation associated to a radial Hamiltonian.

On the other hand, starting with a Talagrand inequality it is possible to derive a logarithmic Sobolev inequality as a consequence of the so called HWI inequality, which relates entropy $(\mathrm{H})$, Wasserstein distance $(\mathrm{W})$ and Fisher information (I). However, this requires an additional geometric assumption on the space. For example, in the Riemannian setting it is sufficient to assume that the reference measure $\mu$ satisfies the Bakry-Emery [2] curvature-dimension inequality $C D(R, \infty)$ with the constant $R>-K$; see [4]. In the more general setting of metric measure spaces we show that this is guaranteed by the assumption that the entropy functional on the Wasserstein space is weakly displacement convex. The notion of weak displacement convexity is defined in the work of Lott and Villani [21]. See also [28] and [29] for questions related to the Ricci curvature in metric measure spaces.

There exist also links to the concentration of measure phenomenon. For instance, Gozlan [13] shows that, in a general metric setting, the dimension-free concentration of measure is equivalent to the Talagrand inequality. In a subsequent paper [15] Gozlan et al. prove that, under curvature assumptions, Gaussian concentration implies the quadratic logarithmic Sobolev inequality.

To summarize our results we denote the $q$-logarithmic Sobolev inequality by $q$-LSI. We also introduce a notion of a $p$-Talagrand inequality, $p$ - $T$, where $p \geq 2$ and $q \leq 2$ are conjugates so that $1 / p+1 / q=1$. We prove that

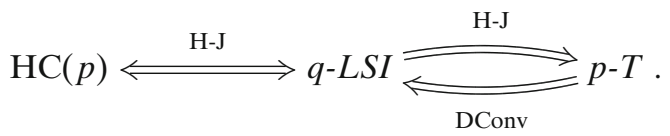


The left-hand side of the diagram represents the hypercontractivity of the infimumconvolution semigroup associated to the exponent $p$, H-J means that the implication is obtained via validity of the Hamilton-Jacobi equation, and DConv stands for the weak displacement convexity of the entropy functional.

The paper is organised as follows. In Section 2 we list some of the important properties of the infimum-convolution semigroup. In Section 3 we establish the equivalence on the left-hand side of the above diagram, provided that the HamiltonJacobi equation is satisfied. (It is the case e.g. when the measure $\mu$ is doubling and supports a local Poincaré inequality.) In Section 4 we consider the relation between the $q$-logarithmic Sobolev inequality and the $p$-Talagrand inequality. Again assuming that the Hamilton-Jacobi equation is satisfied on $X$, we show that the $q$-logarithmic Sobolev inequality implies the $p$-Talagrand inequality. The converse implication holds under the assumption of the weak displacement convexity of the entropy functional on the Wasserstein space of probability measures on $X$. For the reader's convenience Section 5 provides an account of the infimum-convolution semigroup on proper length spaces. The final section is for remarks and further questions. We also indicate here an application of our results by using a recent result of Inglis and Papageorgiou [17] on the logarithmic Sobolev inequality in the subRiemannian setting of the Heisenberg group.

\section{Preliminaries, The Hamilton-Jacobi Equation}

Let $(X, d)$ be a metric space. We say that $d$ is a length metric, if for all $x, y \in X$ we have

$$
d(x, y)=\operatorname{inflength}(\gamma),
$$

where the infimum is taken over all paths that connect $x$ and $y$. Notice, that if $X$ is proper, i.e. its closed and bounded sets are compact, then the infimum is attained and the space is, in fact, geodesic [1].

We remind the reader that a Borel measure $\mu$ is doubling, if the measure of any open ball is positive and finite, and if there exists a constant $c_{d} \geq 1$ such that

$$
\mu(B(x, 2 r)) \leq c_{d} \mu(B(x, r))
$$

for all $x \in X$ and $r>0$. Here $B(x, r)$ denotes an open ball of radius $r$ centered in $x$.

If $f$ is a real-valued Lipschitz function on $X$, we write

$$
\operatorname{lip} f(x)=\liminf _{r \rightarrow 0} \sup _{d(x, y)<r} \frac{|f(x)-f(y)|}{r}
$$

for every $x \in X$.

Let $1 \leq p<\infty$. We say that $(X, d, \mu)$ satisfies a local $(1, p)$-Poincaré inequality (see, for example, [18]) if there exists $1 \leq L<\infty$ and $C>0$, such that for all Lipschitz functions $f$ we have

$$
f_{B(x, r)}\left|f-f_{B(x, r)}\right| d \mu \leq C r\left(f_{B(x, L r)}(\operatorname{lip} f)^{p} d \mu\right)^{1 / p}
$$


for all $x \in X$ and $r>0$. Here we wrote

$$
f_{B(x, r)}=f_{B(x, r)} f d \mu=\frac{1}{\mu(B(x, r))} \int_{B(x, r)} f d \mu .
$$

We remind the reader that if $\mu$ is doubling and the metric space is complete, the above definition coincides with the a priori stronger definition involving upper gradients; see [18] and [19].

Throughout the paper we assume that $d$ is a length metric and $(X, d)$ is proper. Without further notice all measures on $(X, d)$ will be Borel probability measures. We will later impose further assumptions on the space when they are needed.

\subsection{Metric Gradient and Hamilton-Jacobi Equation in Geodesic Spaces}

Consider a function $f: X \times \mathbb{R}_{+} \rightarrow \mathbb{R}$. We define the so called metric gradient of $f$ with respect to the variable $x \in X$ at a point $\left(x_{0}, t\right) \in X \times \mathbb{R}_{+}$as

$$
|\nabla f|\left(x_{0}, t\right):=\limsup _{x \rightarrow x_{0}} \frac{\left|f(x, t)-f\left(x_{0}, t\right)\right|}{d\left(x, x_{0}\right)} .
$$

For an arbitrary function this could be infinite, but if $f$ is Lipschitz continuous in the $x$ variable, the metric gradient $|\nabla f|\left(x_{0}, t\right)$ is always finite. However, it turns out that for the Hamilton-Jacobi equation in metric spaces one should consider a slightly different notion of a gradient. Following the lines in [22], we introduce the so called metric subgradient of $f$ defined as

$$
\left|\nabla^{-} f\right|\left(x_{0}, t\right):=\limsup _{x \rightarrow x_{0}} \frac{\left[f(x, t)-f\left(x_{0}, t\right)\right]_{-}}{d\left(x, x_{0}\right)}=\limsup _{x \rightarrow x_{0}} \frac{\left[f\left(x_{0}, t\right)-f(x, t)\right]_{+}}{d\left(x, x_{0}\right)},
$$

where $a_{+}=\max (a, 0)$ and $a_{-}=\max (-a, 0)$. Notice, that

$$
\left|\nabla^{-} f\right|\left(x_{0}, t\right) \leq|\nabla f|\left(x_{0}, t\right)
$$

and $\left|\nabla^{-} f\right|\left(x_{0}, t\right)$ vanishes if $f(\cdot, t)$ has a local minimum at $x_{0}$. In fact, the metric subgradient indicates that the local variation of $f(\cdot, t)$ takes into account only values less than $f\left(x_{0}, t\right)$.

In analogy to the Euclidean case (see, for example, Evans [11]) the initial-value problem for the Hamilton-Jacobi equation in a geodesic space can be defined as

$$
\left\{\begin{aligned}
\frac{\partial}{\partial t} u(x, t)+H\left(\left|\nabla^{-} u\right|(x, t)\right) & =0 & & \text { in } X \times \mathbb{R}_{+} \\
u(x, t) & =g(x) & & \text { on } X \times\{t=0\} .
\end{aligned}\right.
$$

Throughout the paper we assume that the initial data $g: X \rightarrow \mathbb{R}$ is Lipschitz continuous and the function $H: \mathbb{R}_{+} \rightarrow \mathbb{R}_{+}$is convex, superlinear and satisfies the condition $H(0)=0$. Here $H$ is called the Hamiltonian, and in the Euclidean case a standard example for such a function is $x \mapsto \frac{1}{\alpha}|x|^{\alpha}$ for a real $\alpha>1$. 
The corresponding Hopf-Lax formula (or the infimum-convolution) is defined by

$$
Q_{t} g(x)=\inf _{y \in X}\left[t L\left(\frac{d(x, y)}{t}\right)+g(y)\right],
$$

where $L: \mathbb{R}_{+} \rightarrow \mathbb{R}_{+}$is simply the one-dimensional Legendre transform of $H$ defined by

$$
L(u)=\sup _{v \in \mathbb{R}_{+}}\{u v-H(v)\}, u \in \mathbb{R}_{+} .
$$

Notice, that by standard results the one-dimensional Legendre transformation $L$ is increasing, convex, superlinear and satisfies $L(0)=0$. Moreover,

$$
H(w)=\max _{v \in \mathbb{R}_{+}}\{w v-L(v)\}
$$

We remind the reader that in the Euclidean case the Hopf-Lax formula provides a Lipschitz-continuous solution to the Hamilton-Jacobi equation [11]. This has been generalized to the case of the Heisenberg group [23] (see also [10]) and to the present metric setting setting by [22] for quadratic Hamiltonians. We will show, that under further assumptions on the space this holds also in the metric setting for general Hamiltonians. Namely, we prove the following theorem in Section 5. Notice, that here $\mu$ needs not to be a probability measure.

\section{Theorem 2.1}

(i) The infimum in Eq. 2.2 is attained.

(ii) For $0 \leq s<t$ we have the semigroup property

$$
Q_{t} g(x)=\min _{y \in X}\left[(t-s) L\left(\frac{d(x, y)}{t-s}\right)+Q_{s} g(y)\right]
$$

for all $x \in X$.

(iii) For all $x \in X, Q_{t} g(x)$ is non-increasing in $t$.

(iv) $(x, t) \mapsto Q_{t} g(x)$ is in $\operatorname{Lip}\left(X \times \mathbb{R}_{+}\right)$. If $g$ is only bounded and measurable then $Q_{t} g(x)$ is Lipschitz as a function of $x$ for $t>0$.

(v) For all $x \in X, u(x, t)=Q_{t} g(x)$ solves Eq. 2.1 for a.e. $t>0$.

(vi) For every $x \in X$ and $t>0$

$$
\liminf _{s \rightarrow 0^{+}} \frac{Q_{t+s} g(x)-Q_{t} g(x)}{s} \geq-H\left(\left|\nabla^{-} Q_{t} g\right|(x)\right) .
$$

(vii) If $(X, d, \mu)$ supports a local Poincaré inequality and $\mu$ is doubling, then

$$
\limsup _{s \rightarrow 0^{+}} \frac{Q_{t+s} g(x)-Q_{t} g(x)}{s} \leq-H\left(\left|\nabla^{-} Q_{t} g\right|(x)\right)
$$

for all $t>0$ and $\mu-$ a.e. $x \in X$.

(viii) If $(X, d, \mu)$ supports a local Poincaré inequality and $\mu$ is doubling, $u(x, t)=$ $Q_{t} g(x)$ solves Eq. 2.1 for all $t>0$ and for $\mu$-a.e. $x \in X$. 


\section{Logarithmic Sobolev Inequalities and Hypercontractivity of the Hamilton-Jacobi Semigroup}

\subsection{Logarithmic Sobolev Inequality}

The $q$-logarithmic-Sobolev inequality is a quantitative expression of the fact that the entropy of a function is dominated by the $q$-norm of its gradient. The entropy functional for an integrable, non-negative function $h: X \rightarrow \mathbb{R}_{+}$is defined by

$$
\operatorname{Ent}_{\mu}(h)=\int_{X} h \log h d \mu-\int_{X} h d \mu \log \int_{X} h d \mu .
$$

Definition 1 If $K>0$ and $1<q \leq 2$ we say that $(X, d, \mu)$ satisfies a $q$-log-Sobolev inequality with a constant $K, q-L S I(K)$, if for any Lipschitz function $f$ we have

$$
\operatorname{Ent}_{\mu}\left(|f|^{q}\right) \leq(q-1)\left(\frac{q}{K}\right)^{q-1} \int_{X}\left|\nabla^{-} f\right|^{q} d \mu .
$$

Notice, that for $q>2$ it is not possible to have Eq. 3.2, as for $f=1+\varepsilon g$, where $\varepsilon \rightarrow 0$, the left-hand side behaves like $\varepsilon^{2}$ where as the right-hand side like $\varepsilon^{q}$; see [3]. Notice also, that Corollary 3.2 in [3] provides an example of a measure that satisfies Eq. 3.2.

\subsection{Hypercontractivity of the Hamilton-Jacobi Semigroup}

The equivalence between the hypercontractivity of the quadratic Hamilton-Jacobi semigroup and the logarithmic Sobolev inequality in $\mathbb{R}^{n}$ is established in [4], and our approach follows the same lines.

Let $\mu$ be a probability measure on the Borel sets of $\mathbb{R}^{n}$. We will denote by $\|\cdot\|_{p}, p \geq 1$, the $L^{p}$-norm with respect to $\mu$. Bobkov et al. [4] have shown that a measure $\mu$ which is absolutely continuous with respect to the Lebesgue measure satisfies the classical logarithmic Sobolev inequality with constant $\rho$ if and only if the Hamilton-Jacobi semigroup $Q_{t}$ associated to the quadratic inf-convolution operator is hypercontractive, i.e. we have

$$
\left\|e^{Q_{t} f}\right\|_{a+\rho t} \leq\left\|e^{f}\right\|_{a}
$$

for every bounded measurable function $f$ on $\mathbb{R}^{n}$, every $t \geq 0$ and every $a \in \mathbb{R}$. The strategy of the proof, going back to Gross, consists of studying the monotonicity properties of the left hand side of Eq. 3.3 by differentiating with respect to $t$.

\subsection{Hypercontractivity and Log-Sobolev Inequality}

In this section we prove the equivalence between the $q$-logarithmic Sobolev inequality and the hypercontractivity of the corresponding Hamilton-Jacobi semigroup. To state our result we impose additional conditions on the space $X$ which guarantee that the infimum-convolution $Q_{t} f$ solves the Hamilton-Jacobi equation for a Lipschitz initial-value function $f$. We consider the Hamilton-Jacobi equation on $X$ with the Hamiltonian $H(v)=v^{q} / q$, which corresponds to $L(u)=u^{p} / p$. 
Theorem 3.1 Suppose that $(X, d, \mu)$ supports a local $(1, s)$-Poincaré inequality for some $s \geq 1$, and $\mu$ is doubling. Furthermore, assume that $(X, d, \mu)$ satisfies the $q$ logarithmic Sobolev inequality with some constant $K$, and that $a, \rho>0$ are related by the inequality

$$
a^{2-q} K^{q-1} \geq \rho(q-1) .
$$

Then for every bounded measurable function $f$ on $X$ and every $t \geq 0$

$$
\left\|e^{Q_{t} f}\right\|_{a+\rho t} \leq\left\|e^{f}\right\|_{a} .
$$

Conversely, if Eq. 3.5 holds for all $t \geq 0$, then the q-logarithmic Sobolev inequality, $q$-LSI $\left(K_{0}\right)$, holds on $X$ with a constant $K_{0}$ which satisfies Eq. 3.4 with an equality.

Proof Let $F(t)=\left\|e^{Q_{t} f}\right\|_{\lambda(t)}$ with $\lambda(t)=a+\rho t, t>0$. For all $t>0, \frac{\partial}{\partial t} Q_{t} f(x)$ exists. Hence, $F(t)$ is differentiable at every point $t>0$, and we get

$$
\lambda^{2}(t) F(t)^{\lambda(t)-1} F^{\prime}(t)=\rho \operatorname{Ent}_{\mu}\left(e^{\lambda(t)} Q_{t} f\right)+\int_{X} \lambda^{2}(t) \frac{\partial}{\partial t} Q_{t} f e^{\lambda(t)} Q_{t} f d \mu .
$$

Since $\frac{\partial}{\partial t} Q_{t} f(x)=-\left|\nabla^{-} Q_{t} f(x)\right|^{q} / q \mu$-a.e. in $X$ by Theorem 2.1 (vii), we have

$$
\lambda^{2}(t) F(t)^{\lambda(t)-1} F^{\prime}(t)=\rho \operatorname{Ent}_{\mu}\left(e^{\lambda(t) Q_{t} f}\right)-\lambda^{2}(t) \int_{X} \frac{\left|\nabla^{-} Q_{t} f\right|^{q}}{q} e^{\lambda(t)} Q_{t} f d \mu .
$$

Since $Q_{t} f(x)$ is Lipschitz continuous, we can apply the $q$-logarithmic Sobolev inequality to $e^{\lambda(t)} Q_{t} f$ to deduce that $F^{\prime}(t) \leq 0$ for all $t>0$. Since $F(t)$ is continuous it is non-increasing.

To prove the converse, consider a Lipschitz continuous function $f$. Then Eq. 3.5 implies $F^{\prime}(0) \leq 0$. The Hamilton-Jacobi equation implies

$$
\left.\frac{\partial}{\partial t} Q_{t} f(x)\right|_{t=0}=-\left|\nabla^{-} f(x)\right|^{q} / q
$$

$\mu$-a.-e. in $X$. Thus regarding Eq. 3.6 at $t=0$, we get

$$
\rho \operatorname{Ent}_{\mu}\left(e^{a f}\right) \leq a^{2} \int_{X} e^{a f} \frac{\left|\nabla^{-} f\right|^{q}}{q} d \mu .
$$

By setting $e^{a f}=g^{q}$ this leads to the $K_{0}$-logarithmic Sobolev inequality, where $K_{0}$ satisfies Eq. 3.4 with an equality.

Remark 1 The hypercontractivity of the infimum convolution semigroup holds only for $q \leq 2$.

Proof Indeed, suppose that $q>2$ and consider a bounded non-negative function $f$ with $\operatorname{essup}_{X} f>\int_{X} f d \mu$. Fix a small $\delta>0$.

Since $q>2$, it is possible to choose $t \rightarrow \infty, \varepsilon \rightarrow 0$ so that $\varepsilon^{q-1} t=\delta$ and $\varepsilon t \rightarrow \infty$. Directly from the definition one can check that the scaling property of $Q_{t}$, namely

$$
Q_{t}(\varepsilon f)(x)=\varepsilon\left(Q_{\varepsilon^{q-1} t} f\right)(x)
$$

holds for all $x \in X$ and $t, \varepsilon>0$. 
Then we get from Eq. 3.5 that

$$
\left\|e^{Q_{t}(\varepsilon f)}\right\|_{a+\rho t}^{1 / \varepsilon}=\left\|e^{Q_{\delta} f}\right\|_{(a+\rho t) \varepsilon} \leq\left\|e^{\varepsilon f}\right\|_{a}^{1 / \varepsilon}=\left\|e^{f}\right\|_{a \varepsilon},
$$

whence

$$
e^{\operatorname{essup}_{X}\left(Q_{\delta} f\right)} \leq e^{\int_{X} f d \mu} .
$$

Letting $\delta \rightarrow 0$ we obtain a contradiction.

\section{Talagrand and Logarithmic Sobolev Inequalities}

\subsection{Wasserstein Distance and the Talagrand Inequality}

Let $1 \leq p<\infty$. The $p$-Wasserstein distance between two probability measures on $X$ is defined as

$$
W_{p}(\mu, v)=\left(\inf \iint \frac{1}{p} d(x, y)^{p} d \pi(x, y)\right)^{1 / p},
$$

where the infimum is taken over all probability measures $\pi$ on $X \times X$ with marginals $\mu$ and $\nu$. By the Monge-Kantorovitch dual characterization, see [27], we can write

$$
W_{p}(\mu, v)^{p}=\sup \left[\int_{X} g d v-\int_{X} f d \mu\right],
$$

where the supremum is taken over all pairs $(f, g)$ of bounded measurable functions such that for all $x$ and $y$ we have

$$
g(x) \leq f(y)+\frac{d(x, y)^{p}}{p} .
$$

Recall that the entropy functional for an integrable, non-negative function was defined in Eq. 3.1 in the previous section.

Definition 2 Let $p \geq 2$. We say that $(X, d, \mu)$ satisfies the $p$-Talagrand inequality with a constant $K, p$-Tal $(K)$, if for any probability measure $v \ll \mu$ on $X$ there holds

$$
W_{p}(v, \mu)^{p} \leq \frac{1}{K} \operatorname{Ent}_{\mu}\left(\frac{d v}{d \mu}\right)
$$

Let us mention that our definition differs from the standard version of the Talagrand inequality defined for $1 \leq p \leq 2$, namely

$$
W_{p}(\nu, \mu)^{2} \leq \frac{1}{K} \operatorname{Ent}_{\mu}\left(\frac{d v}{d \mu}\right)
$$

which has been widely studied in the literature, see e.g. [31, Chapter 22]. As we shall show in Theorem 4.1 below, the version (4.3) is equivalent to the appropriate $q$ logarithmic Sobolev inequality.

Notice, that if $d \nu / d \mu$ is of the form $1+\varepsilon g$ where $\varepsilon \rightarrow 0$, then $\operatorname{Ent}_{\mu}(d \nu / d \mu)$ is of order $\varepsilon^{2}$, whereas $W_{p}(\nu, \mu)^{p}$ is typically of order $\varepsilon^{p}$ as the following example shows. 
Let $(M, v o l)$ be a smooth compact connected Riemannian manifold and let $\mu$ and $v$ be two probability measures absolutely continuous with respect to $\mathrm{vol}$, considered as elements of the Wasserstein space of probability measures on $M$ with quadratic distance $W_{2}$. It is known (see [26]) that there is a unique geodesic $\mu_{\varepsilon}$ (with respect to $W_{2}$ ) in the Wasserstein space that joins $\mu$ and $v$. Moreover, the measure is transported along the geodesics in $M$ in the following way. There exists a family of maps $\left\{F_{\varepsilon}\right\}_{\varepsilon \in[0,1]}: M \rightarrow M$ such that $\mu_{\varepsilon}=\left(F_{\varepsilon}\right)_{*} \mu_{0}$. More precisely, for almost all $m \in M, F_{\varepsilon}(m)=\exp _{m}(-\varepsilon \nabla \phi(m))$ for a certain Lipschitz continuous function $\phi$ on $M$ with an almost everywhere defined Hessian (see [9]). It follows that for small $\varepsilon$ we have

$$
\mu_{\varepsilon}(d m)=\mu_{0}(d m)(1+\varepsilon \Delta \phi(m)+o(\varepsilon)) .
$$

Consider the coupling $\left(\mathrm{Id}, F_{\varepsilon}\right)_{*} \mu_{0}$ of $\mu_{0}$ and $\mu_{\varepsilon}$. Then

$$
\begin{aligned}
W_{p}\left(\mu_{0}, \mu_{\varepsilon}\right)^{p} & =\inf _{\pi} \int_{M \times M} \frac{d(x, y)^{p}}{p} d \pi(x, y) \\
& \leq \int_{M \times M} \frac{d(x, y)^{p}}{p} d\left(\left(\mathrm{Id}, F_{\varepsilon}\right)_{*} \mu_{0}\right)(x, y) \\
& =\int_{M} \frac{d\left(m, F_{\varepsilon}(m)\right)^{p}}{p} d \mu_{0}(m) \\
& =\varepsilon^{p} \int_{M} \frac{|\nabla \phi(m)|^{p}}{p} d \mu_{0}(m) .
\end{aligned}
$$

Thus Eq. 4.3 does not hold for $1 \leq p<2$.

\subsection{The Dual Formulation of the Talagrand Inequality}

To establish a connection between the Talagrand and the log-Sobolev inequality, we have to consider the dual formulation of the Talagrand inequality using the Hamilton-Jacobi semigroup. For an arbitrary function $f$ on $X$, consider the infimum convolution (2.2) with Lagrangian $L(u)=u^{p} / p$, namely

$$
Q_{t} f(x)=\inf _{y \in X}\left[\frac{d(x, y)^{p}}{p t^{p-1}}+f(y)\right],
$$

and write $Q f$ for $Q_{1} f$. Following [5], we notice that by the Monge-Kantorovitch duality (4.1) and Eq. 4.2, the $p$-Talagrand inequality is equivalent to

$$
\int_{X}\left(Q f-\int_{X} f d \mu\right) \frac{d \nu}{d \mu} d \mu \leq \frac{1}{K} \operatorname{Ent}_{\mu}\left(\frac{d \nu}{d \mu}\right),
$$

for every bounded function $f$. Define two functions: $\psi_{0}:=K\left(Q f-\int_{X} f d \mu\right)$ and $\phi:=\frac{d v}{d \mu}$. Recall that by the variational characterization of the entropy

$$
\operatorname{Ent}_{\mu}(\phi)=\sup _{\int_{X} e^{\psi} d \mu \leq 1} \int_{X} \psi \phi d \mu .
$$

Indeed, the left-hand side is smaller than or equal to the right-hand side by definition. The converse inequality results from Jensen's inequality applied to the convex function $x \mapsto x \log x$ and the probability measure $e^{\psi} d \mu / \int_{X} e^{\psi} d \mu$. 
Since Eq. 4.4 holds for every choice of $\frac{d v}{d \mu}$, it is therefore equivalent to $\int_{X} e^{\psi_{0}} d \mu \leq$ 1, i.e.

$$
\int_{X} e^{K Q f} d \mu \leq e^{K \int_{X} f d \mu}
$$

The latter inequality is known as the dual form of the $p$-Talagrand inequality.

\subsection{Talagrand and Log-Sobolev Inequality}

In order to state the main result of this section we need to recall one more concept, the notion of displacement convexity from [21]. Recall that for $p \in[1, \infty)$ the space $P_{p}(X)$ of Borel probability measures on a compact length space $X$ with the Wasserstein distance $W_{p}$ is itself a compact length space, see [21, Remark 2.8]. If $v$ is a probability measure which is absolutely continuous with respect to $\mu$, we define the entropy functional $U_{\mu}$ on $P_{p}(X)$ by

$$
U_{\mu}(v)=\int_{X} \frac{d \nu}{d \mu} \log \left(\frac{d v}{d \mu}\right) d \mu=\operatorname{Ent}_{\mu}\left(\frac{d \nu}{d \mu}\right) .
$$

Following [21], we say that it is weakly displacement convex if for all $v_{0}, v_{1} \in P_{p}(X)$, there is some Wasserstein geodesic $\left\{v_{t}\right\}_{t \in[0,1]}$ from $v_{0}$ to $v_{1}$ along which

$$
U_{\mu}\left(v_{t}\right) \leq t U_{\mu}\left(v_{1}\right)+(1-t) U_{\mu}\left(v_{0}\right) .
$$

Notice also, that in the Riemannian setting, Villani considers a version of Theorem 4.1 with a different choice of Lagrangian; see [31, Theorem 22.28].

Theorem 4.1 Let $2 \geq q>1$ and $p \geq 2$ be its conjugate, so that $1 / p+1 / q=1$.

(i) Let $(X, d, \mu)$ satisfy the $p$-Talagrand inequality with some constant $K>0$, and assume that $X$ is compact. If the entropy functional $U_{\mu}(\cdot)$ is weakly displacement convex then $(X, d, \mu)$ also satisfies the q-logarithmic Sobolev inequality with the constant $K p^{-p}$.

(ii) Suppose that $(X, d, \mu)$ supports a local $(1, s)$-Poincaré inequality for some $s \geq 1$, and $\mu$ is doubling. Then, if $(X, d, \mu)$ satisfies the q-logarithmic Sobolev inequality with some constant $K>0$, then it also satisfies the $p$-Talagrand inequality with the same constant.

Proof Consider a probability measure $v$ on $X$ with a positive Lipschitz continuous density function $f$ with respect to $\mu$. Then from [21, Proposition 3.36] it can be easily deduced that

$$
U_{\mu}(v) \leq \int_{X \times X} \frac{\left|\nabla^{-} f\left(x_{0}\right)\right|}{f\left(x_{0}\right)} d\left(x_{0}, x_{1}\right) d \pi\left(x_{0} x_{1}\right),
$$

where $\pi$ is the optimal coupling of $(\nu, \mu)$. Applying the Hölder inequality on the right-hand side gives

$$
U_{\mu}(\nu) \leq p^{1 / p} W_{p}(\mu, v)\left(\int_{X} \frac{\left|\nabla^{-} f\left(x_{0}\right)\right|^{q}}{f^{q-1}\left(x_{0}\right)} d \mu\left(x_{0}\right)\right)^{1 / q} .
$$


Hence the $p$-Talagrand inequality implies

$$
U_{\mu}(v)=\operatorname{Ent}_{\mu}(f) \leq\left(\frac{p}{K}\right)^{q / p} \int_{X} \frac{\left|\nabla^{-} f\right|^{q}}{f^{q-1}} d \mu .
$$

Replacing $f$ with $|g|^{q}$ we arrive at the $q$-logarithmic Sobolev inequality, $q$ - $L S I\left(K p^{-p}\right)$, with the desired constant. This proves (i).

To prove (ii) we follow the idea in [22]. We consider the Hamilton-Jacobi equation on $X$ with the Hamiltonian $H(v)=v^{q} / q$, which corresponds to $L(u)=u^{p} / p$ and the associated semigroup (2.2) $Q f=Q_{1} f$. From the Talagrand inequality in its dual formulation (4.5) it follows that it is sufficient to show that

$$
\int_{X} e^{K Q f} d \mu \leq e^{K \int_{X} f d \mu}
$$

for every continuous bounded function $f$. Set, for some $n \geq 1$,

$$
\phi(t)=\frac{1}{K t^{n}} \log \left(\int_{X} e^{K t^{n} Q_{t} f} d \mu\right) .
$$

Since $f$ is bounded, we know that $Q_{t} f$ is bounded uniformly in $t$. Thus

$$
\int_{X} e^{K t^{n} Q_{t} f} d \mu=1+K t^{n} \int_{X} Q_{t} f d \mu+O\left(t^{2 n}\right),
$$

and

$$
\phi(t)=\int_{X} Q_{t} f d \mu+O\left(t^{n}\right)
$$

Since $Q_{t} f \rightarrow f$ as $t \rightarrow 0^{+}$, we have by the dominated convergence theorem that

$$
\lim _{t \rightarrow 0^{+}} \phi(t)=\int_{X} f d \mu .
$$

Therefore, our goal is to prove that $\phi(1) \leq \lim _{t \rightarrow 0^{+}} \phi(t)$. For this, it suffices to prove that $\phi(t)$ is non-increasing in $t$. Let us fix $t \in(0,1]$. For $s>0$, we have

$$
\begin{aligned}
\frac{\phi(t+s)-\phi(t)}{s}= & \frac{1}{s}\left(\frac{1}{K(t+s)^{n}}-\frac{1}{K t^{n}}\right) \log \int_{X} e^{K(t+s)^{n} Q_{t+s} f} d \mu \\
& +\frac{1}{K t^{n} s}\left(\log \int_{X} e^{K(t+s)^{n} Q_{t+s} f} d \mu-\log \int_{X} e^{K t^{n} Q_{t} f} d \mu\right) .
\end{aligned}
$$

As $s \rightarrow 0^{+}$, the first term on the right-hand side converges to

$$
-\frac{n}{K t^{n+1}} \log \left(\int_{X} e^{K t^{n} Q_{t} f} d \mu\right) .
$$

The limit of the second term, provided it exists, is

$$
\frac{1}{K t^{n}} \frac{1}{\int_{X} e^{K t^{n} Q_{t} f} d \mu} \lim _{s \rightarrow 0^{+}}\left[\frac{1}{s}\left(\int_{X} e^{K(t+s)^{n} Q_{t+s} f} d \mu-\int_{X} e^{K t^{n} Q_{t} f} d \mu\right)\right] .
$$


The expression in brackets can be written as

$$
\int_{X}\left(\frac{e^{K(t+s)^{n} Q_{t+s} f}-e^{K t^{n} Q_{t+s} f}}{s}\right) d \mu+\int_{X}\left(\frac{e^{K t^{n} Q_{t+s} f}-e^{K t^{n} Q_{t} f}}{s}\right) d \mu .
$$

The first term in Eq. 4.6 has the form $e^{K t^{n} Q_{t+s} f}\left(e^{K\left(n t^{n-1} s+o(s)\right) Q_{t+s} f}-1\right) / s$ so it converges to $\left(e^{K t^{n} Q_{t} f}\right) K n t^{n-1} Q_{t} f$ as $s \rightarrow 0^{+}$. By the dominated convergence theorem the first integral in Eq. 4.6 thus converges to

$$
\int_{X} K n t^{n-1} Q_{t} f e^{K t^{n} Q_{t} f} d \mu .
$$

Let us now consider the second term of Eq. 4.6. By Theorem 2.1 (vi) and (vii), for $\mu$-a.e. $x \in X$ we have

$$
Q_{t+s} f(x)=Q_{t} f(x)-s\left(\frac{\left|\nabla^{-} Q_{t} f(x)\right|^{q}}{q}+o(1)\right),
$$

and therefore

$$
\lim _{s \rightarrow 0^{+}} \frac{e^{K t^{n} Q_{t+s} f}-e^{K t^{n} Q_{t} f}}{s}=-K t^{n} e^{K t^{n} Q_{t} f} \frac{\left|\nabla^{-} Q_{t} f\right|^{q}}{q} .
$$

On the other hand, as $Q_{(\cdot)} g(\cdot)$ is Lipschitz on $X \times \mathbb{R}_{+}, Q_{t+s} f=Q_{t} f+O(s)$ holds uniformly on $X$. Since $Q_{t} f(x)$ is uniformly bounded in $x$, we deduce that

$$
\frac{e^{K t^{n} Q_{t+s} f}-e^{K t^{n} Q_{t} f}}{s}=O(1)
$$

as $s \rightarrow 0^{+}$. In view of Eqs. 4.7 and 4.8 we apply the dominated convergence theorem to compute the limit of the second integral in Eq. 4.6, that is,

$$
\lim _{s \rightarrow 0^{+}} \int_{X}\left(\frac{e^{K t^{n} Q_{t+s} f}-e^{K t^{n} Q_{t} f}}{s}\right) d \mu=-K t^{n} \int_{X} \frac{\left|\nabla^{-} Q_{t} f\right|^{q}}{q} e^{K t^{n} Q_{t} f} d \mu .
$$

In summary, we have

$$
\begin{aligned}
\lim _{s \rightarrow 0^{+}}\left[\frac{\phi(t+s)-\phi(t)}{s}\right]= & \frac{1}{K t^{n+1} \int_{X} e^{K t^{n} Q_{t} f} d \mu} \\
\times[ & -n \log \left(\int_{X} e^{K t^{n} Q_{t} f} d \mu\right) \int_{X} e^{K t^{n} Q_{t} f} d \mu \\
& +\int_{X} n K t^{n} Q_{t} f e^{K t^{n} Q_{t} f} d \mu \\
& \left.-\int_{X} K t^{n+1} \frac{\left|\nabla^{-} Q_{t} f\right|^{q}}{q} e^{K t^{n} Q_{t} f} d \mu\right] .
\end{aligned}
$$

Recall that for $q \in(1,2]$, the $q$-logarithmic Sobolev inequality with constant $K$ states that for every Lipschitz function $g$ on $X$

$$
\operatorname{Ent}_{\mu}\left(|g|^{q}\right) \leq(q-1)\left(\frac{q}{K}\right)^{q-1} \int_{X}\left|\nabla^{-} g\right|^{q} d \mu .
$$


Set $n=1 /(q-1)$. Applying Eq. 4.10 with $g=\exp \left(K t^{n} Q_{t} f / q\right)$ shows that Eq. 4.9 is non-positive, and (ii) follows.

Remark 2 Let $p=q=2$. In the setting of Riemannian manifolds, i.e. when $X=$ $(M, v o l)$, the displacement convexity in the first part of Theorem 4.1 is verified if the reference measure $\mu=e^{-V}$ vol, with $\mu(M)=1$ and $V \in C^{2}(M)$, satisfies the curvature-dimension $C D(0, \infty)$ inequality; see [21].

Remark 3 Let us note that the equivalence between transport and logarithmic Sobolev type inequalities does not hold without curvature assumptions. A counterexample was found in [7].

\section{Solutions to Hamilton-Jacobi Equation}

Proof of Theorem 2.1 (i) Fix $x \in X$ and $t>0$. Notice, that by choosing $y=x$ in Eq. 2.2 we get $Q_{t} g(x) \leq g(x)$.

Let $\left(y_{n}\right)$ be a minimizing sequence in Eq. 2.2 and assume first that it is bounded. Since $X$ is proper there exists $y_{0} \in X$ and a subsequence $\left(y_{n_{k}}\right)$ such that $y_{n_{k}} \rightarrow y_{0}$, whence the continuity of $L$ and $g$ imply that

$$
Q_{t} g(x)=\lim _{k \rightarrow \infty}\left\{t L\left(\frac{d\left(x, y_{n_{k}}\right)}{t}\right)+g\left(y_{n_{k}}\right)\right\}=t L\left(\frac{d\left(x, y_{0}\right)}{t}\right)+g\left(y_{0}\right) .
$$

On the other hand, if $\lim _{n \rightarrow \infty} d\left(y_{n}, x\right)=+\infty$, the superlinearity of $L$ implies that for any $M>0$ we have

$$
L\left(\frac{d\left(x, y_{n}\right)}{t}\right) \geq M \frac{d\left(x, y_{n}\right)}{t}
$$

for $n$ large enough. Multiplying the above inequality by $t$ and adding $g\left(y_{n}\right)$ on both sides we get

$$
t L\left(\frac{d\left(x, y_{n}\right)}{t}\right)+g\left(y_{n}\right) \geq M d\left(x, y_{n}\right)+g\left(y_{n}\right) \geq(M-\operatorname{lip}(g)) d\left(x, y_{n}\right)-|g(x)|,
$$

since $g$ is Lipschitz. Choosing $M:=\operatorname{lip}(g)+1$ we obtain

$$
t L\left(\frac{d\left(x, y_{n}\right)}{t}\right)+g\left(y_{n}\right) \geq d\left(x, y_{n}\right)-|g(x)|,
$$

which implies that

$$
\lim _{n \rightarrow \infty} t L\left(\frac{d\left(x, y_{n}\right)}{t}\right)+g\left(y_{n}\right)=\infty,
$$

which is a contradiction. Hence $\left(y_{n}\right)$ is bounded and the infimum in Eq. 2.2 is attained.

Proof of Theorem 2.1 (ii) Fix $q \in X$. By (i) there exists a $v \in X$ such that

$$
Q_{s} g(q)=s L\left(\frac{d(v, q)}{s}\right)+g(v)
$$


Set $\tau:=\frac{s}{t}, \sigma:=\frac{t-s}{t}$, and use the monotonicity and convexity of $L$ to obtain

$$
\begin{aligned}
L\left(\frac{d(v, p)}{t}\right) & \leq L\left(\tau \frac{d(v, q)}{\tau t}+\sigma \frac{d(q, p)}{\sigma t}\right) \leq \tau L\left(\frac{d(v, q)}{\tau t}\right)+\sigma L\left(\frac{d(q, p)}{\sigma t}\right) \\
& =\frac{s}{t} L\left(\frac{d(v, q)}{s}\right)+\frac{t-s}{t} L\left(\frac{d(q, p)}{t-s}\right) .
\end{aligned}
$$

Multiplying the inequality by $t$ and adding $g(v)$ on both sides yields

$$
\begin{aligned}
Q_{t} g(p) \leq t L\left(\frac{d(v, p)}{t}\right)+g(v) & \leq(t-s) L\left(\frac{d(q, p)}{t-s}\right)+s L\left(\frac{d(v, q)}{s}\right)+g(v) \\
& =(t-s) L\left(\frac{d(q, p)}{t-s}\right)+Q_{s} g(q)
\end{aligned}
$$

Since $q \in X$ is arbitrary we obtain

$$
Q_{t} g(p) \leq \min _{q \in X}\left\{(t-s) L\left(\frac{d(q, p)}{t-s}\right)+Q_{s} g(q)\right\} .
$$

Notice, that this does not depend on the fact that $d$ is a length metric.

To show the reverse inequality we use the properties of the geodesic metric $d$. Again by (i) we can choose for $(p, t) \in X \times \mathbb{R}_{+}$such $w \in X$ that minimizes Eq. 2.2. Now, if $q^{\prime} \in X$ is on a length-minimizing path from $p$ to $w$, we have

$$
d(w, p)=d\left(q^{\prime}, p\right)+d\left(w, q^{\prime}\right)
$$

and for a given $\sigma, \tau>0$ such that $\sigma+\tau=1$ we can find $q^{\prime} \in X$ satisfying

$$
d\left(q^{\prime}, p\right)=\tau d(w, p), \quad d\left(w, q^{\prime}\right)=\sigma d(w, p) .
$$

By setting $\sigma=\frac{s}{t}$, and consequently $\tau=\frac{t-s}{t}$, we obtain

$$
\frac{d(w, p)}{t}=\frac{t}{t-s} \frac{d\left(q^{\prime}, p\right)}{t}=\frac{t}{s} \frac{d\left(w, q^{\prime}\right)}{t}
$$

and, moreover,

$$
L\left(\frac{d(w, p)}{t}\right)=L\left(\frac{d\left(q^{\prime}, p\right)}{t-s}\right)=L\left(\frac{d\left(w, q^{\prime}\right)}{s}\right) .
$$

This implies that

$$
t L\left(\frac{d(w, p)}{t}\right)=(t-s) L\left(\frac{d\left(q^{\prime}, p\right)}{t-s}\right)+s L\left(\frac{d\left(w, q^{\prime}\right)}{s}\right) .
$$


Finally, we add $g(w)$ on both sides of Eq. 5.1 and deduce

$$
\begin{aligned}
Q_{t} g(p) & =t L\left(\frac{d(w, p)}{t}\right)+g(w)=(t-s) L\left(\frac{d\left(q^{\prime}, p\right)}{t-s}\right)+s L\left(\frac{d\left(w, q^{\prime}\right)}{s}\right)+g(w) \\
& \geq(t-s) L\left(\frac{d\left(q^{\prime}, p\right)}{t-s}\right)+\min _{v \in X}\left\{s L\left(\frac{d\left(v, q^{\prime}\right)}{s}\right)+g(v)\right\} \\
& =(t-s) L\left(\frac{d\left(q^{\prime}, p\right)}{t-s}\right)+Q_{s} g\left(q^{\prime}\right) \\
& \geq \min _{q \in X}\left\{(t-s) L\left(\frac{d(q, p)}{t-s}\right)+Q_{s} g(q)\right\} .
\end{aligned}
$$

Proof of Theorem 2.1 (iii) By (ii), for a fixed $p \in X$ we have

$$
\begin{aligned}
Q_{t} g(p) & =\min _{q \in X}\left\{(t-s) L\left(\frac{d(q, p)}{t-s}\right)+Q_{s} g(q)\right\} \\
& \leq(t-s) L(0)+Q_{s} g(p)=Q_{s} g(p)
\end{aligned}
$$

by choosing $p=q$ and using $L(0)=0$.

Proof of Theorem 2.1 (iv) In fact, we will prove that

$$
\operatorname{lip}\left(Q_{(\cdot)} g(\cdot)\right) \leq \max \{\operatorname{lip}(g), H(\operatorname{lip}(g))\}
$$

where lip stands for the Lipschitz constant of the corresponding function (of one or two variables). On $X \times \mathbb{R}_{+}$we assume the canonical product metric

$$
d_{X \times \mathbb{R}_{+}}((x, t),(y, s))=d(x, y)+|s-t| .
$$

We recall that by Rademacher's theorem the Lipschitz continuity of $Q_{(\cdot)} g(\cdot)$ implies differentiability of $Q_{(\cdot)} g(x)$ a.e. in the $t$ variable.

We shall fix $t>0$ and show the Lipschitz continuity of $x \rightarrow Q_{t} g(x)$ first. Let $x, \xi \in$ $X$ be arbitrary, and choose a minimizing $y_{0}$ in Eq. 2.2 for $(\xi, t)$. By the Lipschitz continuity of $g$ we get

$$
\begin{aligned}
Q_{t} g(x)-Q_{t} g(\xi) & \leq t L\left(\frac{d(q, x)}{t}\right)+g(q)-t L\left(\frac{d\left(\xi, y_{0}\right)}{t}\right)-g\left(y_{0}\right) \\
& \leq t\left[L\left(\frac{d(q, x)}{t}\right)-L\left(\frac{d\left(\xi, y_{0}\right)}{t}\right)\right]+\operatorname{lip}(g) d\left(q, y_{0}\right)
\end{aligned}
$$

for any $q \in X$.

Assume first that $d\left(x, y_{0}\right) \geq d(x, \xi)$. Choose $q$ on the minimizing geodesic from $y_{0}$ to $x$ such that $d\left(q, y_{0}\right)=d(x, \xi)$, and hence $d(x, q) \leq d\left(\xi, y_{0}\right)$. Since $L$ is increasing this with Eq. 5.2 implies that

$$
\begin{aligned}
Q_{t} g(x)-Q_{t} g(\xi) & \leq t\left[L\left(\frac{d(q, x)}{t}\right)-L\left(\frac{d\left(\xi, y_{0}\right)}{t}\right)\right]+\operatorname{lip}(g) d\left(q, y_{0}\right) \\
& \leq \operatorname{lip}(g) d(x, \xi) .
\end{aligned}
$$


Assume then that $d\left(x, y_{0}\right)<d(x, \xi)$. Since $Q_{t} g(x) \leq g(x)$, choose $q=x$ in Eq. 5.2 to obtain

$$
\begin{aligned}
Q_{t} g(x)-Q_{t} g(\xi) & \leq g(x)-t L\left(\frac{d\left(\xi, y_{0}\right)}{t}\right)-g\left(y_{0}\right) \\
& \leq \operatorname{lip}(g) d\left(x, y_{0}\right)-t L\left(\frac{d\left(\xi, y_{0}\right)}{t}\right) \leq \operatorname{lip}(g) d(x, \xi) .
\end{aligned}
$$

The two estimates now lead to

$$
Q_{t} g(x)-Q_{t} g(\xi) \leq \operatorname{lip}(g) d(x, \xi)
$$

for all $x, \xi \in X$, and simply interchanging $p$ and $\xi$ implies the desired Lipschitz continuity.

We now turn to the Lipschitz continuity of $t \rightarrow Q_{t} g(x)$. With no loss of generality we assume $0<s<t$. Since $u$ is non-increasing in $t$ we have $Q_{t} g(x)-Q_{s} g(x) \leq 0$. By (ii) we get

$$
\begin{aligned}
Q_{t} g(x) & \left.=Q_{s} g(x)+\min _{q \in X}\left\{(t-s) L\left(\frac{d(x, q)}{t-s}\right)+Q_{s} g(q)-Q_{s} g(x)\right)\right\} \\
& \geq Q_{s} g(x)+\min _{q \in X}\left\{(t-s) L\left(\frac{d(x, q)}{t-s}\right)-\operatorname{lip}(g) d(x, q)\right\} \\
& \geq Q_{s} g(x)+(t-s) \min _{v \in \mathbb{R}_{+}}\{L(v)-\operatorname{lip}(g) v\} \\
& =Q_{s} g(x)-(t-s) H(\operatorname{lip}(g)),
\end{aligned}
$$

where $v=d(x, q) /(t-s)$. This shows that

$$
\left|Q_{t} g(x)-Q_{s} g(x)\right| \leq H(\operatorname{lip}(g))|t-s| .
$$

Now the Lipschitz continuity in both variables imply

$$
\begin{aligned}
\left|Q_{t} g(p)-Q_{s} g(\xi)\right| & \leq\left|Q_{t} g(p)-Q_{s} g(p)\right|+\left|Q_{s} g(p)-Q_{s} g(\xi)\right| \\
& \leq \max \{H(\operatorname{lip}(g)), \operatorname{lip}(g)\}(|t-s|+d(p, \xi)) .
\end{aligned}
$$

The fact that $Q_{t} g(x)$ is Lipschitz as a function of $x$ when $g$ is bounded and measurable and $t>0$, follows from [22].

Proof of Theorem $2.1(v)$ We show that

$$
\frac{\partial}{\partial t} u(x, t)+H\left(\left|\nabla^{-} u\right|(x, t)\right) \leq 0
$$

holds for every $x \in X$ and a.e. $t \in \mathbb{R}_{+}$for $u(x, t)=Q_{t} g(x)$. The converse inequality follows from (vi).

Fix $x \in X$ and let $t \in \mathbb{R}_{+}$be a point of differentiability of $u(x, \cdot)$. If $\left|\nabla^{-} u\right|(x, t)=0$, Eq. 5.3 reduces to $u_{t}(x, t) \leq 0$ since $H(0)=0$. This clearly holds since $u(x, \cdot)$ is nonincreasing.

We can thus assume that $\left|\nabla^{-} u\right|(x, t)>0$, and there exists a sequence $x_{n} \rightarrow x$ for which $u\left(x_{n}, t\right)<u(x, t)$ and

$$
\left|\nabla^{-} u\right|(x, t)=\lim _{n \rightarrow \infty} \frac{u(x, t)-u\left(x_{n}, t\right)}{d\left(x_{n}, x\right)} .
$$


For the moment, consider any positive sequence $\left(h_{n}\right)$ with $h_{n} \rightarrow 0$. By the semi-group property (ii) we get

$$
u\left(x, t+h_{n}\right)=\min _{y \in X}\left\{h_{n} L\left(\frac{d(x, y)}{h_{n}}\right)+u(y, t)\right\} \leq h_{n} L\left(\frac{d\left(x, x_{n}\right)}{h_{n}}\right)+u\left(x_{n}, t\right),
$$

which implies that

$$
\frac{u\left(x, t+h_{n}\right)-u(x, t)}{h_{n}} \leq-\left[\frac{u(x, t)-u\left(x_{n}, t\right)}{h_{n}}-L\left(\frac{d\left(x, x_{n}\right)}{h_{n}}\right)\right] .
$$

Since $H(w)=\max _{v \in \mathbb{R}_{+}}\{w v-L(v)\}$ for all $w \in \mathbb{R}_{+}$, for each $n$ it is possible to choose $h_{n}>0$ such that

$$
H\left(\frac{u(x, t)-u\left(x_{n}, t\right)}{d\left(x_{n}, x\right)}\right)=\frac{u(x, t)-u\left(x_{n}, t\right)}{h_{n}}-L\left(\frac{d\left(x, x_{n}\right)}{h_{n}}\right)
$$

holds. Furthermore, it is easy to see directly from Eq. 5.5 that $x_{n} \rightarrow x$ implies $h_{n} \rightarrow 0$.

Finally, combining Eqs. 5.4 and 5.5 we obtain

$$
\frac{u\left(x, t+h_{n}\right)-u(x, t)}{h_{n}}+H\left(\frac{u(x, t)-u\left(x_{n}, t\right)}{d\left(x_{n}, x\right)}\right) \leq 0 .
$$

As $x_{n} \rightarrow x$ and $h_{n} \rightarrow 0$, letting $n \rightarrow \infty$ gives us Eq. 5.3.

Proof of Theorem 2.1 (vi) Let us fix $x \in X$ and $t \in \mathbb{R}_{+}$. Since $(x, t) \mapsto Q_{t} g(x)$ is a Lipschitz function, the limes inferior in Eq. 2.3 is finite and we can choose a positive sequence $\left(h_{n}\right)$ such that $h_{n} \rightarrow 0$ and

$$
\liminf _{s \rightarrow 0^{+}} \frac{Q_{t+s} g(x)-Q_{t} g(x)}{s}=\lim _{n \rightarrow \infty} \frac{Q_{t+h_{n}} g(x)-Q_{t} g(x)}{h_{n}} .
$$

Next, applying the semigroup property we can write

$$
Q_{t+h_{n}} g(x)=\min _{y \in X}\left\{h_{n} L\left(\frac{d(x, y)}{h_{n}}\right)+Q_{t} g(y)\right\} .
$$

For each $n$ we choose a point $y_{n} \in X$ for which the minimum is attained. The superlinearity of $L$ implies that $y_{n} \rightarrow x$.

As $Q_{t} g(x)$ is decreasing in $t$, we have $Q_{t+h_{n}} g(x) \leq Q_{t} g(x)$, and hence

$$
Q_{t} g\left(y_{n}\right) \leq h_{n} L\left(\frac{d(x, y)}{h_{n}}\right)+Q_{t} g\left(y_{n}\right) \leq Q_{t} g(x) .
$$

Since $H(w)=\max _{v \in \mathbb{R}_{+}}\{w v-L(v)\}$ we have $H(w)+L(v) \geq w v$ for all $w, v \in \mathbb{R}_{+}$. Together with Eq. 5.8 this implies that

$$
H\left(\frac{Q_{t} g(x)-Q_{t} g\left(y_{n}\right)}{d\left(x, y_{n}\right)}\right)+L\left(\frac{d\left(x, y_{n}\right)}{h_{n}}\right) \geq \frac{Q_{t} g(x)-Q_{t} g\left(y_{n}\right)}{h_{n}},
$$

and we have

$$
L\left(\frac{d\left(x, y_{n}\right)}{h_{n}}\right)+\frac{Q_{t} g\left(y_{n}\right)-Q_{t} g(x)}{h_{n}} \geq-H\left(\frac{\left[Q_{t} g(x)-Q_{t} g\left(y_{n}\right)\right]_{+}}{d\left(x, y_{n}\right)}\right) .
$$


Together with Eq. 5.7 this implies

$$
\begin{aligned}
\frac{Q_{t+h_{n}} g(x)-Q_{t} g(x)}{h_{n}} & =\frac{1}{h_{n}}\left(h_{n} L\left(\frac{d\left(x, y_{n}\right)}{h_{n}}\right)+Q_{t} g\left(y_{n}\right)-Q_{t} g(x)\right) \\
& \geq-H\left(\frac{\left[Q_{t} g(x)-Q_{t} g\left(y_{n}\right)\right]_{+}}{d\left(x, y_{n}\right)}\right) .
\end{aligned}
$$

Letting now $n \rightarrow \infty$ and using Eq. 5.6 we obtain

$$
\begin{aligned}
\liminf _{s \rightarrow 0^{+}} \frac{Q_{t+s} g(x)-Q_{t} g(x)}{s} & \geq \limsup _{n \rightarrow \infty}\left(-H\left(\frac{\left[Q_{t} g(x)-Q_{t} g\left(y_{n}\right)\right]_{+}}{d\left(x, y_{n}\right)}\right)\right) \\
& \geq-H\left(\left|\nabla^{-} Q_{t} g\right|(x)\right) .
\end{aligned}
$$

Notice, that if $u(x, t)=Q_{t} g(x)$, and $t$ is a point of differentiability of $t \rightarrow u(x, t)$ for a fixed $x$, then it follows from (vi) that

$$
u_{t}(x, t)+H\left(\left|\nabla^{-} u\right|(x, t)\right) \geq 0 .
$$

Since $u$ is Lipschitz-continuous, the above inequality holds for all $x \in X$ and a.e. $t \in \mathbb{R}_{+}$. This finishes the proof of $(\mathrm{v})$.

Proof of Theorem 2.1 (vii) We prove (vii) along the lines in [22, Theorem 2.5 (vii)]. If $\left|\nabla^{-} Q_{t} g\right|(x)=0$ the statement is trivial since $Q_{t} g(x)$ is non-increasing in $t$. Let $t>0$ be fixed and assume that $\left|\nabla^{-} Q_{t} g\right|(x)>0$. Define $f(x):=Q_{t} g(x)$ and fix a real number $\alpha>0$. By the semi-group property (ii) we get for $s>0$

$$
\begin{aligned}
\frac{Q_{t} g(x)-Q_{t+s} g(x)}{s} & =\frac{1}{s} \sup _{y \in X}\left[f(x)-f(y)-s L\left(\frac{d(x, y)}{s}\right)\right] \\
& \geq \sup _{y \in S_{\alpha s}(x)}\left[\frac{f(x)-f(y)}{d(x, y)} \alpha-L(\alpha)\right],
\end{aligned}
$$

where $S_{r}(x)$ denotes the sphere of radius $r$ around $x$. Write

$$
\psi(r)=\sup _{y \in S_{r}(x)} \frac{f(x)-f(y)}{d(x, y)} .
$$

It is shown in [22] that $\liminf _{r \rightarrow 0^{+}} \psi(r)=\left|\nabla^{-} f\right|(x)$ a.e. on $X$. Thus

$$
\liminf _{s \rightarrow 0^{+}} \frac{Q_{t} g(x)-Q_{t+s} g(x)}{s} \geq\left|\nabla^{-} Q_{t} g\right|(x) \alpha-L(\alpha) .
$$

Maximizing the above inequality over $\alpha>0$ we obtain that

$$
\liminf _{s \rightarrow 0^{+}} \frac{Q_{t} g(x)-Q_{t+s} g(x)}{s} \geq H\left(\left|\nabla^{-} Q_{t} g\right|(x)\right),
$$

which is equivalent to the statement of the proposition.

Finally, (vi) and (vii) together prove (viii). 


\section{Applications, Comments and Questions}

In the work by Bobkov and Ledoux [6] some $q$-logarithmic Sobolev inequalities were derived from the Prekopa-Leindler inequality.

A large class of geodesic metric measure spaces for which the Poincaré inequality holds-and our results apply_are the Carnot-Carathéodory geometries; see, for example, [18] and [16]. A case of particular interest within this class is the class of Carnot groups where many fundamental results of Euclidean analysis hold. In this setting, Hamilton-Jacobi equations have already been considered by Manfredi and Stroffolini [23], see also [10]. It would be interesting to characterize measures for which an appropriate Log-Sobolev inequality holds on Carnot-Carathéodory spaces. In the Euclidean setting results in this direction were obtained by Barthe and Kolesnikov [3]. In the case of the first Heisenberg group $\mathbb{H}$, Inglis and Papageorgiou showed in the recent paper [17] that the measure

$$
\mu_{p}(d x)=\frac{e^{-\beta d^{p}(x)}}{\int_{\mathbb{H}} e^{-\beta d^{p}(x)} d x} d x
$$

satisfies the $q$-Log-Sobolev inequality. Here $\beta>0$ is an arbitrary number, $p \geq 2$ is the conjugate exponent to $q, d x$ is the Lebesgue measure and $d(x)$ is the subRiemannian Carnot-Carathéodory distance on $\mathbb{H}$. In order to apply our results, one has to note that for smooth functions $f: \mathbb{H} \rightarrow \mathbb{R}$ the norm of the sub-Riemannian gradient $|\nabla f(x)|$ from [17] and our metric subgradient $\left|\nabla^{-} f(x)\right|$ coincide for $\mu_{p}$ a.e. $x$ for which $|\nabla f(x)|>0$. For Lipschitz continuous functions this follows from Pansu's differentiability theorem [16].

Therefore the $q$-Log-Sobolev inequality according to Definition 1 holds in this setting. Applying our results one obtains the validity of the $p$-Talagrand inequality and hypercontractivity of the Hamilton-Jacobi semigroup in the setting of the Heisenberg group equipped with the sub-Riemannian metric and the above probability measure $\mu_{p}$.

Furthermore, it would be interesting to see whether the results of this paper hold in the more general class of metric measure spaces satisfying the so-called Lip-lip condition. To be precise, let us recall from [20] that a metric measure space $(X, d, \mu)$ satisfies the Lip-lip condition if there exists a constant $L \geq 1$ with the property that if $f: X \rightarrow \mathbb{R}$ is a Lipschitz function then

$$
\operatorname{Lip} f(x) \leq L \cdot \operatorname{lip} f(x), \text { for } \mu \text {-a.e. } x \in X,
$$

where $\operatorname{Lip} f(x)$ and $\operatorname{lip} f(x)$ are the local Lipschitz numbers of $f$ at $x$ defined as

$$
\begin{aligned}
& \operatorname{Lipf}(x)=\limsup _{r \rightarrow 0} \sup _{y \in B(x, r)} \frac{|f(x)-f(y)|}{r}, \\
& \operatorname{lip} f(x)=\liminf _{r \rightarrow 0} \sup _{y \in B(x, r)} \frac{|f(x)-f(y)|}{r} .
\end{aligned}
$$

Let us recall that Keith proved in [20] that if a metric measure space $(X, d, \mu)$, where $\mu$ is doubling, satisfies the Lip-lip condition then $X$ supports a measurable differentiable structure in the sense of Cheeger [8]. Keith also proved that if the doubling metric measure space $(X, d, \mu)$ satisfies the Poincaré inequality then the Lip-lip condition is satisfied. It is also clear that the Lip-lip condition is more general 
than the Poincaré inequality, for example a positive measure Cantor set in the Euclidean space satisfies this condition but does not support a Poincaré inequality.

Finally, it would be interesting to prove a variant of Hopf-Lax formula for the solution of the Hamilton-Jacobi equation, i.e. Theorem 2.1 for the case of geodesic spaces satisfying the Lip-lip condition. It is clear that statements (i) through (vi) will hold true without modification. Furthermore, it is reasonable to expect that statement (vii) will be replaced by

$$
\limsup _{s \rightarrow 0^{+}} \frac{Q_{t+s} g(x)-Q_{t} g(x)}{s} \leq-H\left(\frac{\left|\nabla^{-} Q_{t} g\right|(x)}{L^{\prime}}\right)
$$

for all $t>0$ and $\mu$-a.e. $x \in X$ and for some absolute constant $L^{\prime} \geq 1$ depending on $(X, d, \mu)$.

The statements of the other results of the paper concerning the circle of equivalences of Talagrand, Log-Sobolev inequalities and hypercontractivity would then follow (with possibly adjusted constants) along the same lines as in the case of metric spaces satisfying a Poincaré inequality.

Acknowledgement The authors are grateful to Cedric Villani for his valuable comments and observations concerning the manuscript.

\section{References}

1. Ambrosio, L., Tilli, P.: Selected topics on "analysis in metric spaces". Appunti dei Corsi Tenuti da Docenti della Scuola. [Notes of Courses Given by Teachers at the School]. Scuola Normale Superiore, Pisa (2000)

2. Bakry, D., Émery, M.: Diffusions hypercontractives. In: Séminaire de Probabilités, XIX, 1983/84, Lecture Notes in Mathematics, vol. 1123, pp. 177-206. Springer, Berlin (1985)

3. Barthe, F., Kolesnikov, A.V.: Mass transport and variants of the logarithmic Sobolev inequality. J. Geom. Anal. 18(4), 921-979 (2008)

4. Bobkov, S.G., Gentil, I., Ledoux, M.: Hypercontractivity of Hamilton-Jacobi equations. J. Math. Pures Appl. (9), 80(7), 669-696 (2001)

5. Bobkov, S.G., Götze, F.: Exponential integrability and transportation cost related to logarithmic Sobolev inequalities. J. Funct. Anal. 163(1), 1-28 (1999)

6. Bobkov, S.G., Ledoux, M.: From Brunn-Minkowski to Brascamp-Lieb and to logarithmic Sobolev inequalities. Geom. Funct. Anal. 10(5), 1028-1052 (2000)

7. Cattiaux, P., Guillin, A.: On quadratic transportation cost inequalities. J. Math. Pures Appl. 86(9), 341-361 (2006)

8. Cheeger, J.: Differentiability of Lipschitz functions on metric measure spaces. Geom. Funct. Anal. 9(3), 428-517 (1999)

9. Cordero-Erausquin, D., McCann, R.J., Schmuckenschläger, M.: A Riemannian interpolation inequality à la Borell, Brascamp and Lieb. Invent. Math. 146(2), 219-257 (2001)

10. Dragoni, F.: Metric Hopf-Lax formula with semicontinuous data. Discrete Contin. Dyn. Syst. 17(4), 713-729 (2007)

11. Evans, L.C.: Partial Differential Equations, Graduate Studies in Mathematics, vol. 19. American Mathematical Society, Providence (1998)

12. Gentil, I., Malrieu, F.: Equations de Hamilton-Jacobi et inégalités entropiques généralisées. C. R. Math. Acad. Sci. Paris 335(5), 437-440 (2002)

13. Gozlan, N.: A characterization of dimension free concentration in terms of transportation inequalities. Ann. Probab. 37(6), 2480-2498 (2009)

14. Gozlan, N., Roberto, C., Samson, P.-M.: A new characterization of Talagrand's transportentropy inequalities and applications. Ann. Probab. 39(3), 857-880 (2011)

15. Gozlan, N., Roberto, C., Samson, P.-M.: From concentration to logarithmic Sobolev and Poincaré inequalities. J. Funct. Anal. 260(5), 1491-1522 (2010) 
16. Gromov, M.: Carnot-Carathéodory spaces seen from within. In: Sub-Riemannian Geometry, Progr. Math., vol. 144, pp. 79-323. Birkhäuser, Basel (1996)

17. Inglis, J., Papageorgiou, N.S.: Logarithmic Sobolev inequalities for infinite dimensional Hörmander type generators on the Heisenberg group. Potential Anal. 31(1), 79-102 (2009)

18. Heinonen, J., Koskela, P.: Quasiconformal maps in metric spaces with controlled geometry. Acta Math. 181(1), 1-61 (1998)

19. Keith, S.: Modulus and the Poincaré inequality on metric measure spaces. Math. Z. 245(2), 255292 (2003)

20. Keith, S.: A differentiable structure for metric measure spaces. Adv. Math. 183(2), 271-315 (2004)

21. Lott, J., Villani, C.: Ricci curvature for metric-measure spaces via optimal transport. Ann. Math. 169, 903-991 (2009)

22. Lott, J., Villani, C.: Hamilton-Jacobi semigroup on length spaces and applications. J. Math. Pures Appl. (9) 88(3), 219-229 (2007)

23. Manfredi, J.J., Stroffolini, B.: A version of the Hopf-Lax formula in the Heisenberg group. Commun. Partial Differ. Equ. 27(5-6), 1139-1159 (2002)

24. Marton, K.: A simple proof of the blowing-up lemma. IEEE Trans. Inf. Theory 32(3), 445-446 (1986)

25. Marton, K.: Bounding $\bar{d}$-distance by informational divergence: a method to prove measure concentration. Ann. Probab. 24(2), 857-866 (1996)

26. McCann, R.J.: Polar factorization of maps on Riemannian manifolds. Geom. Funct. Anal. 11(3), 589-608 (2001)

27. Rachev, S.T.: The Monge-Kantorovich mass transference problem and its stochastic applications. Theory Probab. Appl. 29(4), 647-676 (1985)

28. Sturm, K.-T.: On the geometry of metric measure spaces. I. Acta Math. 196(1), 65-131 (2006)

29. Sturm, K.-T.: On the geometry of metric measure spaces. II. Acta Math. 196(1), 133-177 (2006)

30. Talagrand, M.: Transportation cost for Gaussian and other product measures. Geom. Funct. Anal. 6(3), 587-600 (1996)

31. Villani, C.: Optimal transport: old and new. Grundlehren der Mathematischen Wissenschaften, vol. 338. Springer, Berlin (2009) 